\title{
Adjuvant Treatment Approach in Primary Apocrine Adenocarcinoma Presenting With Axillary Mass: A Case Report
}

Gulcan Bulut ( $\sim$ gulcanbulut07@gmail.com )

Department of Medical Oncology, Defne Hospital, Hatay, Turkey

\section{Sukran Senyurek}

Koc Universitesi

Remziye Eren

Necip Fazıl City Hospital

\section{Case report}

Keywords: Apocrine carcinoma, Occult breast cancer, Radiotherapy, Tamoxifen

Posted Date: August 28th, 2020

DOI: https://doi.org/10.21203/rs.3.rs-64324/v1

License: (1) (i) This work is licensed under a Creative Commons Attribution 4.0 International License.

Read Full License 


\section{Abstract}

Background: Primary apocrine sweat gland adenocarcinoma is a very rare tumor. Apocrine carcinoma is a high incidence of local recurrence and lymph node metastasis. When the location of the tumor is axilla, it should be differentiated from occult breast cancer. Surgery is the first step in primary apocrine cancer treatment. However, there is no clear consensus about adjuvant part of treatment. In this case, we presented a 60-year old female patient with primary apocrine sweat gland carcinoma of the axilla. To our knowledge, this is the first case in the literature to use combined adjuvant radiation therapy and antiestrogen therapy.

Case presentation: A 60-year old female patient presented with a slowly growing mass in the right axilla. The patient was examined by a surgeon and there was suspected to be metastasis from breast cancer diagnosed in September 2017. Axillary localized apocrine carcinoma was differentiated from occult breast cancer by pathological findings. For this reason, the patient was operated only an axillary dissection operation. Operated patient with axillary apocrine carcinoma was treated with radiotherapy. As a result of pathological evaluation of the tumor, tamoxifen was added to the treatment when the hormone receptor was positive.

Conclusions: The pathological features must be evaluated in detail for targeted treatment, which should be ap-plied with a multidisciplinary approach. Based on this case presentation and literature, adjuvant radiotherapy can be recommended to reduce the risk of local recurrence and hormone-therapy to reduce distant recurrence in patients with hormone-receptor positive primary apocrine carcinoma.

\section{Background}

Primary apocrine sweat gland adenocarcinoma is a very rare tumor. It usually emerges in areas of high apocrine gland density such as the axilla and urogenital areas, but may also be seen on the fore head, wrists, ear canals, eye lids, trunk, feet, toes, and fingers. It often presents as painless, indurate, nodules, or plaques and a slow-growing mass, but sometimes it can progress aggressively. Apocrine carcinoma is mostly seen in the 6th decade, with a median age at diagnosis of 67 years and there is a high incidence of local recurrence and lymph node metastasis [1]. The primary treatment is wide local excision with a clear margin of $1-2 \mathrm{~cm}$, with or without axillary lymphadenectomy according to the node clinical status [2]. Adjuvant treatment is controversial, particularly chemotherapy, but adjuvant radiation therapy has been suggested for locally advanced tumors [3]. Anti-estrogen therapy was recommended by Seong et al., but there are no case reports in literature of the use of adjuvant endocrine therapy [3].

In this case, we presented a 60-year old female patient with primary apocrine sweat gland carcinoma of the axilla. To our knowledge, this is the first case in the literature to use combined adjuvant radiation therapy and anti-estrogen therapy.

\section{Case Presentation}


A 60-year old female patient presented with a slowly growing mass in the right axilla. The patient was examined by a surgeon and there was suspected to be metastasis from breast cancer diagnosed in September 2017. The mass in the right axilla was painless, mobile and subcutaneous. No palpable lesion was detected in the bilateral neck lymph nodes and left axilla region and the breast examination was normal. On the initial ultrasonography (USG) examination conglomerate lymph nodes were observed in the right axillary region, the largest being $40 \mathrm{~mm}$ in size. The same findings were seen on bilateral breast magnetic resonance imaging (MRI) (Figure 1). No breast lesion was observed on USG or MRI.

Fine needle aspiration biopsy was performed on the axillary mass. Microscopic examination showed large cytoplasmic, prominent nucleoli and vesicular nucleated cells (Figure 2). As a result of the immunohistochemical examination, pancytoceratine, cytokeratin-7 (CK-7), CEA, gross cystic disease fluid protein-15 (GCFDP-15) and estrogen expression were positive in tumor and CD-68, CK-20, TTF-1 and calretinin were negative (Figure 3). The Cerb-B2 expression and Ki-67 proliferation index were evaluated immunohistochemically. Poor Cerb-B2 expression and 50\% Ki-67 index were detected. The final pathology report was of apocrine cancer and the pathologist recommended evaluation for primary or secondary axillary apocrine cancer. Positron emission tomography (PET/CT) was applied to the patient for the differential diagnosis of metastasis but no metastasis or other primary lesion were detected (Figure 4). When all these results were evaluated, the patient was diagnosed with primary apocrine cancer and surgical excision was recommended. Level I - II axillary dissection was performed by the surgery department. Macroscopic examination of the specimen taken revealed two different conglomerate lymph node materials. The microscopic findings were the same as the first pathological examination and estrogen receptor was $70 \%$ positive.

Adjuvant treatment was planned as radiation therapy and tamoxifen. Adjuvant radiation therapy was started in the supraclavicular and right axillary region in the fourth week after surgery. Radiation therapy was delivered with a total dose of $50 \mathrm{~Gy}$ in 25 fractions (Figure 5). The patient was received Tamoxifen at $20 \mathrm{mg} /$ day after radiation therapy and was planned to using this medication for five years. No local or distant recurrence was detected during a 32-month follow-up period.

\section{Discussion}

The current study is the first case in the literature to have used combined adjuvant hormone therapy and radiotherapy with a long follow-up period without any recurrence.

Primary apocrine cancer is a rare cancer, but it is usually seen in the axillary region. In cases presenting with an axillary mass, differentiation from breast apocrine cancer is important. While breast apocrine cancer is generally a triple negative breast subtype, primary sweat gland apocrine cancer frequently expresses estrogen and progesterone receptors [4,5]. GCFDP - 15 and PAS-positive diastase-resistant material in the cells or luminal positivity is an important immunohistochemical finding that supports a diagnosis of apocrine cancer [6]. However, it is necessary to verify that there is no other organ involvement with clinical imaging to definitively diagnose primary apocrine cancer. 
Surgery is the first step in primary apocrine cancer treatment. However, there is no consensus about the adjuvant part of the treatment. There are cases in the literature where radiotherapy and chemotherapy have been used in adjuvant therapy or followed up after surgery [2,3,7]. Apocrine cancer is resistant to chemotherapy. Radiotherapy is recommended in local or regional advanced disease [2,3]. Evaluation of Cerb-B2 expression is important especially in locally advanced and metastatic patients. Cerb-B2 expression positivity enables the use of targeted therapy [8]. In a retrospective analysis of 186 patients, the median overall survival rate was reported to be 51.5 months for loco-regional disease and 14.5 months for distant metastatic disease [1]. The poor survival and lack of treatment options in stage IV disease indicate the need for effective adjuvant treatment.

\section{Conclusions}

Apocrine cancer is a rare tumor, for which there is no clear consensus about treatment. The pathological features must be evaluated in detail for targeted treatment, which should be applied with a multidisciplinary approach. Based on this case presentation and literature, adjuvant radiotherapy can be recommended to reduce the risk of local recurrence and hormone therapy to reduce distant recurrence in patients with hormone-receptor positive primary apocrine carcinoma.

\section{List Of Abbreviations}

USG: Ultrasonography

MRI: Magnetic resonance imaging

CK-7: Cytokeratin-7

GCFDP-15: Gross cystic disease fluid protein-15

PET/CT: Positron emission tomography

\section{Declarations}

\section{Acknowledgement}

Not applicable

\section{Authors' contributions}

GB supervised the treatment of patient; development of study, wrote manuscript and acted as corresponding author. SS and RE are planned radiotherapy treatment and applied it to patient and also wrote manuscript. All authors take full responsibility for the content of the final paper.

\section{Funding}


Funding information is not applicable

\section{Availability of data and materials}

Not applicable

\section{Ethics approval and consent to participate}

Written informed consent was obtained from the parents prior to publication of this case report, and all procedures performed were approved by the Institutional Review Board for Clinical Research at our university.

\section{Consent for publication}

Written informed consent was obtained from the parents to publish the information, including their photographs. A copy of the written consent is available for review.

\section{Competing interests}

The authors declare that there is no conflict of interest.

\section{References}

1. Hollowell KL, Agle SC, Zervos EE, Fitzgerald TL (2012) Cutaneous apocrine adenocarcinoma: defining epidemiology, outcomes, and optimal therapy for a rare neoplasm. J Surg Oncol 105 (4):415-419. doi:10.1002/jso.22023

2. Chamberlain RS, Huber K, White JC, Travaglino-Parda R (1999) Apocrine gland carcinoma of the axilla: review of the literature and recommendations for treatment. Am J Clin Oncol 22 (2):131-135. doi:10.1097/00000421-199904000-00005

3. Seong MK, Kim EK, Han K, Seol H, Kim HA, Noh WC (2015) Primary apocrine sweat gland carcinomas of the axilla: a report of two cases and a review of the literature. World J Surg Oncol 13:59. doi:10.1186/s12957-015-0473-1

4. Zhao S, Ma D, Xiao Y, Jiang YZ, Shao ZM (2018) Clinicopathologic features and prognoses of different histologic types of triple-negative breast cancer: A large population-based analysis. Eur J Surg Oncol 44 (4):420-428. doi:10.1016/j.ejso.2017.11.027

5. Robson A, Lazar AJ, Ben Nagi J, Hanby A, Grayson W, Feinmesser M, Granter SR, Seed P, Warneke CL, McKee PH, Calonje E (2008) Primary cutaneous apocrine carcinoma: a clinico-pathologic analysis of 24 cases. Am J Surg Pathol 32 (5):682-690. doi:10.1097/PAS.0b013e3181590ba4

6. Paties C, Taccagni GL, Papotti M, Valente G, Zangrandi A, Aloi F (1993) Apocrine carcinoma of the skin. A clinicopathologic, immunocytochemical, and ultrastructural study. Cancer 71 (2):375-381. doi:10.1002/1097-0142(19930115)71:2<375::aid-cncr2820710218>3.0.co;2-4 
7. Zahid R, Soofi ME, Elmalik H, Junejo K (2016) Primary apocrine carcinoma of the axilla in a male patient: a case report. Clin Case Rep 4 (4):344-347. doi:10.1002/ccr3.515

8. Otsuka M, Yamasaki O, Kaji T, Shien T, Iwatsuki K (2016) Metastatic Cutaneous Apocrine Adenocarcinoma Treated With a Combination of Pertuzumab-Based Targeted Therapy and Taxane Chemotherapy: A Case Report. JAMA Dermatol 152 (1):111-113.

doi:10.1001/jamadermatol.2015.2507

\section{Figures}

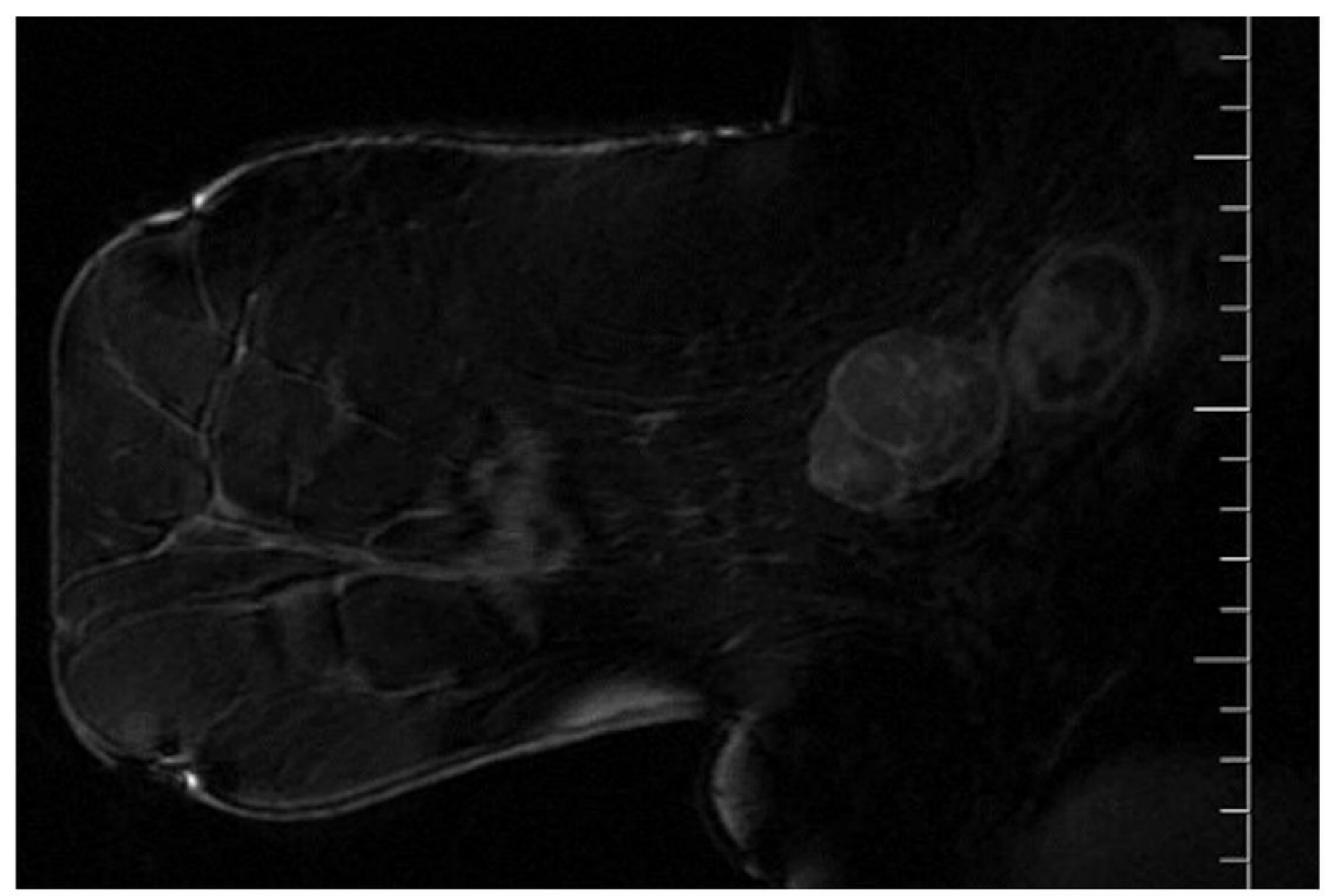

\section{Figure 1}

Conglomerate lymph nodes in the right axillary region, the largest of which is $40 \mathrm{~mm}$ in size, in bilateral breast magnetic resonance imaging (MRI) 


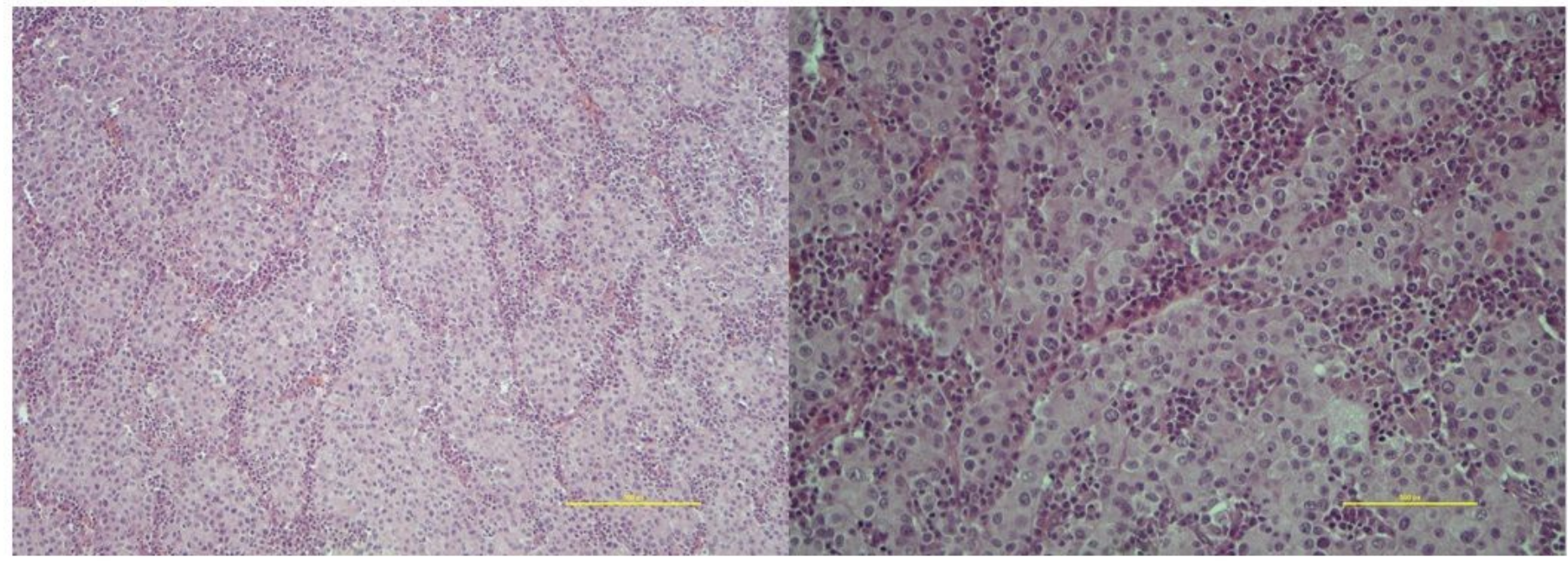

\section{Figure 2}

Tumor layers consisting of cells with granular eosinophilic large cytoplasm and large round nuclei, which completely filled the lymph node, separated by a thin fibrous stroma (Hematoxylin and eosin x 200 and $x$ 400).

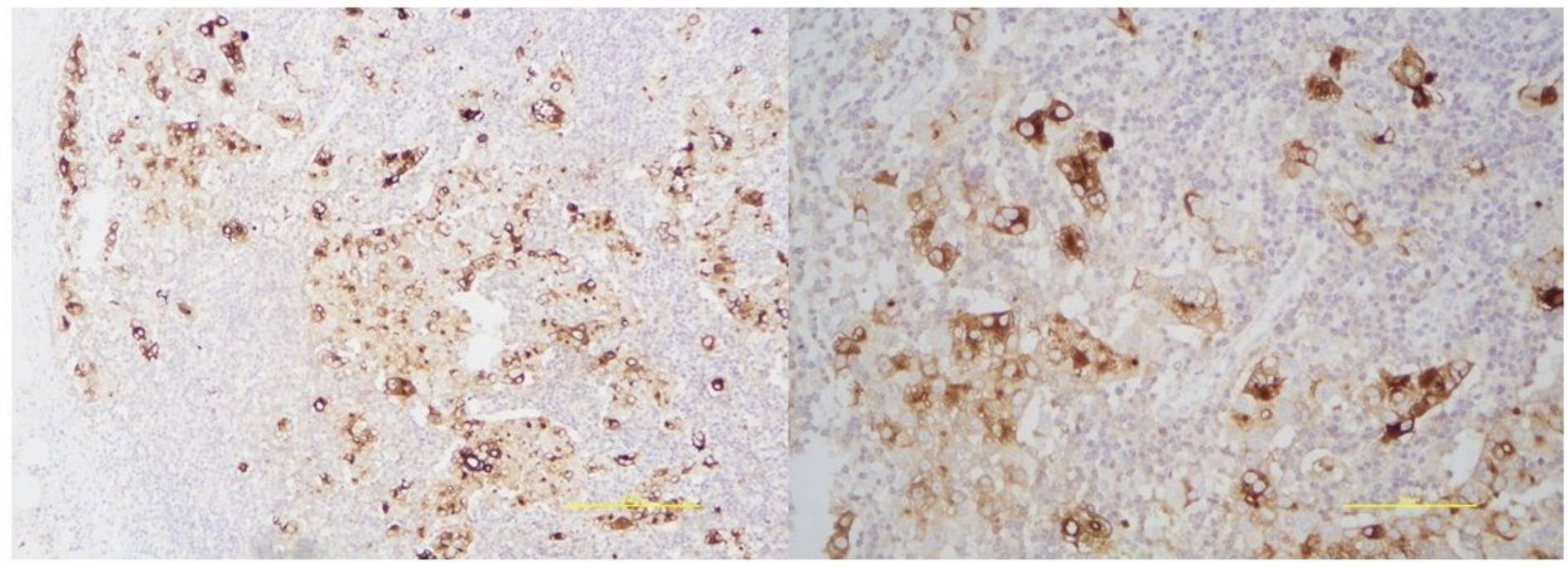

Figure 3

Cytoplasmic positive staining with Gross cystic Disease fluid protein-15 (GCDFP-15) (Hematoxylin and eosin $\times 100$ and $\times 400$ ). 


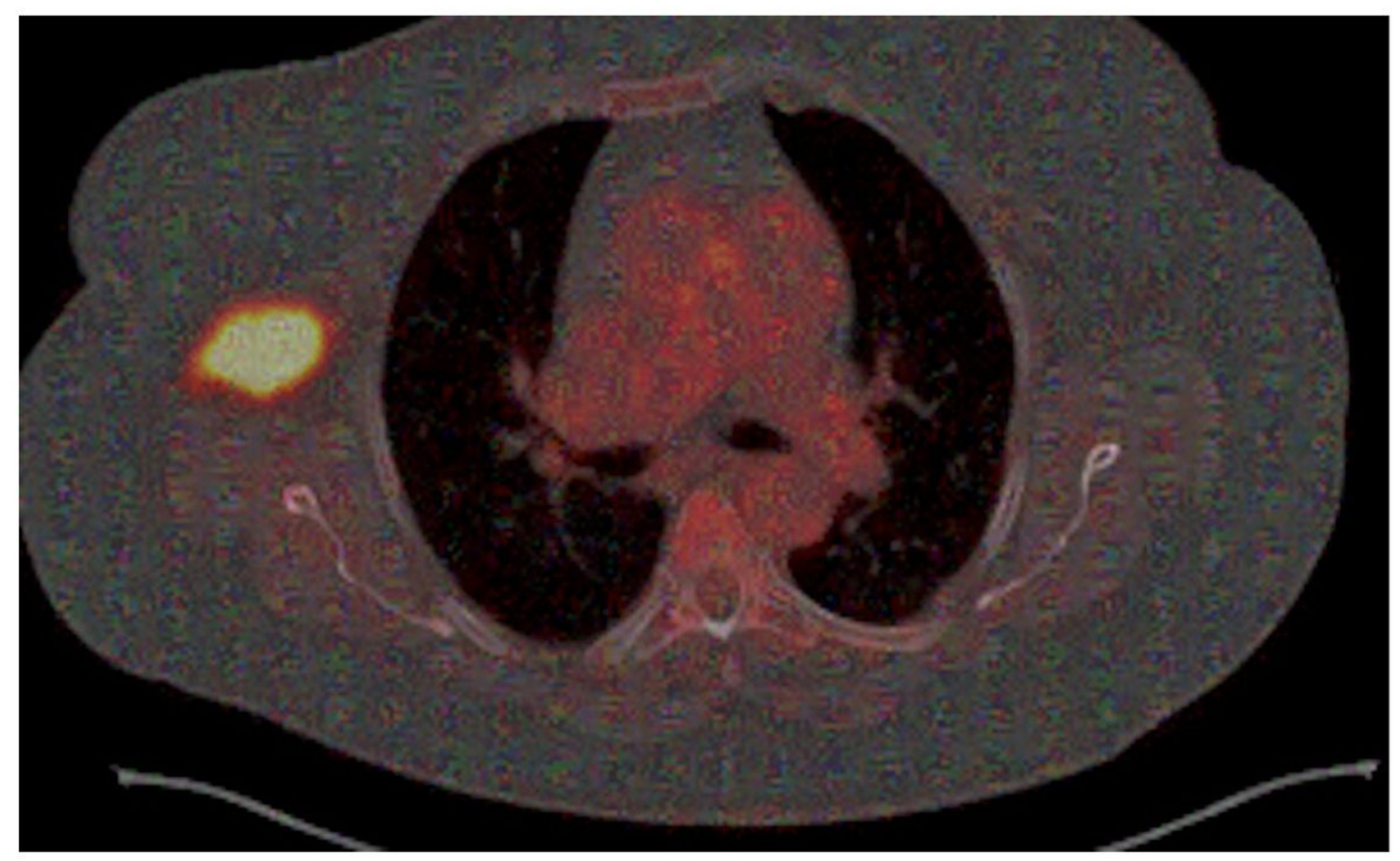

Figure 4

No metastasis or any other primary lesion was detected in positron emission tomography (PET / CT) performed for the differential diagnosis of metastasis. 


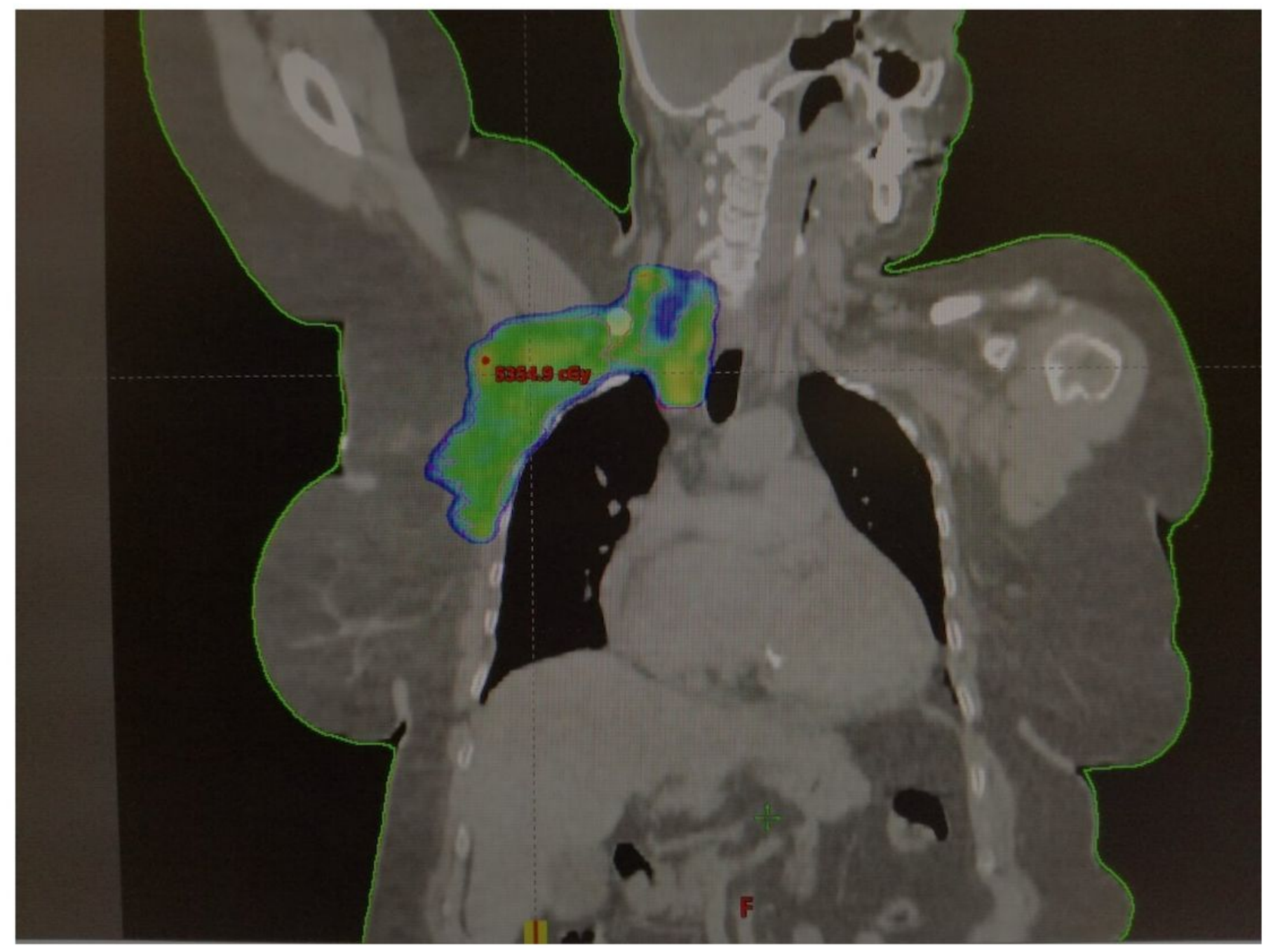

Figure 5

Radiation therapy given in 25 fractions with a total dose of $50 \mathrm{~Gy}$. 